In Cres. Vol. $5 N^{\circ} 1$ : pp. 23-36, 2014

\title{
ESTILO DE VIDA Y FACTORES BIOSOCIOCULTURALES DEL ADULTO MAYOR. URBANIZACIÓN EL CARMEN, CHIMBOTE $^{*}$
}

\author{
LIFESTYLE AND BIOSOCIOCULTURAL FACTORS OF ELDERLY PEOPLE \\ IN THE RESIDENTIAL COMPLEX EL CARMEN, CHIMBOTE
}

\author{
Juana Esther Carranza Benites', \\ Yolanda Rodríguez Núñez ${ }^{2}$
}

\section{RESUMEN}

Este trabajo de investigación es cuantitativo, de corte transversal y diseño descriptivo correlacional. Se realizó con el objetivo de determinar la relación entre el estilo de vida y los factores biosocioculturales del adulto mayor de la urbanización El Carmen, distrito de Chimbote, con una muestra de 242 adultos mayores. Se utilizaron dos instrumentos para la recolección de datos: escala del estilo de vida y el cuestionario sobrefactores biosocioculturales. Los datos fueron procesados con el software SPSS versión 18.0. Para establecer la relación entre las variables de estudio se aplicó la prueba de independencia de criterios Chi cuadrado, con el 95\% de confiabilidad y significancia de $\mathrm{p}<0,05$. Se llegó a las siguientes conclusiones: la mayoría de los adultos mayores presenta un estilo de vida saludable y un porcentaje considerable de estilos de vida no saludable; respecto a los factores biosocioculturales, más de la mitad son de sexo femenino, menos de la mitad presenta grado de instrucción primaria completa e incompleta y percibe de 600 a 1000 soles mensuales; la mayoría profesa la religión católica y son casados; poco más de la mitad son amas de casa. Existe relación estadísticamente significativa entre el estilo de vida con el grado de instrucción en los adultos mayores. No existe relación estadísticamente significativa entre estilos de vida y factores biosocioculturales de edad, sexo, estado civil, ocupación e ingreso económico.

PALABRAS CLAVE: Estilos de vida, factores biosocioculturales, adulto mayor.

* Recibido: 28 de enero del 2014; aprobado: 7 de junio del 2014.

1 Estudiante de Enfermería del VIII ciclo de la Universidad Católica Los Ángeles de Chimbote. Chimbote - Perú.

2 Doctora en Enfermería. Docente investigadora de la Escuela Profesional de Enfermería de la Universidad Católica Los Ángeles de Chimbote. Chimbote - Perú. 


\begin{abstract}
The present research is quantitative, cross-sectional and descriptive correlational design was carried out with the overall objective of determining the relationship between lifestyle factors and the elderly biosocioculturales of urbanization "El Carmen", with a sample of 242 older adults, two instruments were used for data collection: the scale of lifestyle factors and questionnaire biosocioculturales. Data were processed with the software SPSS version 18.0. To establish the relationship between the variables of study applied the test of independence chi-square criteria, with $95 \%$ reliability and significance of $p<0,05$. It reached the following conclusions: Most older adults have a healthy lifestyle and a significant proportion have unhealthy lifestyles. Regarding the reasons biosocioculturales of older adults are: more than half are older people are female. Less than half have completed primary education level and incomplete and receives 600 to 1000 soles a month. Most are Catholic and married status. Just over half are housewives. If there is a statistically significant relationship between lifestyle and cultural factors: Level of education among older adults in the urbanization El Carmen. No statistically significant relationship between lifestyles and biosocioculturales factors of: age, sex, marital status, occupation and income.
\end{abstract}

KEY WORDS: Lifestyle, factors biosocioculturales, senior.

\title{
INTRODUCCIÓN
}

En los países subdesarrollados los estilos de vida poco saludables son los que causan la mayoría de enfermedades. La persona cuyo estilo de vida en sus años previos se caracterizó porque nunca hizo actividad física, su nutrición fue deficiente o tuvo hábitos nocivos, tendrá problemas en su vejez; de ahí la importancia de las actividades y programas de promoción y prevención en la salud.

Para disminuir la incidencia y mortalidad por enfermedad prevenible, es necesario identificar las circunstancias que favorecen el desarrollo y la aceleración de la enfermedad. Es importante, en el intento por controlar este problema, valorar la presencia de riesgos modificables o controlables para luego actuar en ello, considerando ajustes de los estilos de vida de las personas. ${ }^{2}$

Muchos de estos estilos de vida incluyen fundamentalmente una alimentación adecuada, promoción de la actividad física, control del tabaquismo y otras adicciones, ambientes saludables en viviendas y trabajos, conductas sexuales, salud mental y control de tres elementos ligados a la alimentación: presión arterial, colesterol y arteriosclerosis.

A esta realidad no escapa la urbanización El Carmen, que se ubica en la jurisdicción del Puesto de Salud Túpac Amaru, en el distrito de Chimbote, provincia del Santa, ubicada en la parte norte del Perú. Todas las viviendas son de material noble, disponen de los servicios básicos: luz eléctrica, agua y desagüe, 
cuentan con dos instituciones educativas de nivel primario y secundario: Virgen del Carmen y Micaela Bastidas; también existe una parroquia y un parque; asimismo, cuenta con 7 sectores y un campo deportivo. ${ }^{4}$

\section{PROBLEMA}

¿Existe relación entre el estilo de vida y los factores biosocioculturales del adulto mayor de la urbanización El Carmen, Chimbote en el año 2012?

\section{OBJETIVO GENERAL}

Determinar la relación entre el estilo de vida y los factores biosocioculturales del adulto mayor de la Urbanización "El Carmen" de Chimbote. Asimismo, se formularon los siguientes objetivos específicos:

1. Conocer el estilo de vida del adulto mayor de la Urbanización El Carmen, de Chimbote.

2. Identificar los factores biosocioculturales: sexo, religión, grado de instrucción, estado civil, ocupación, ingreso económico.

\section{HIPÓTESIS}

Existiría relación significativa entre el estilo de vida y los factores biosocioculturales del adulto mayor de la urbanización El Carmen, de Chimbote.

\section{JUSTIFICACIÓN}

La realización de la investigación se justifica debido a que el presente estudio dará una visión de cómo estamos y que la clave está en la prevención, en crear conciencia, en simples cambios para mejorar la nutrición e incrementar la actividad física. Hemos de llegar especialmente a comunidades vulnerables, incluidos los trabajadores inmigrantes y las familias. Esta investigación es necesaria para los establecimientos de salud, porque las conclusiones y recomendaciones pueden contribuir a elevar la eficiencia del programa preventivo promocional de la salud del adulto y reducir los factores de riesgo, así como mejorar el estilo de vida de las personas.

\section{METODOLOGÍA}

El estudio es de tipo cuantitativo, de corte transversal, descriptivo correlacional. La población estuvo conformada por 650 personas adultas mayores que residen en la urbanización El Carmen, del distrito de Chimbote, provincia del 
Santa, región Ancash, de los cuales se obtuvo una muestra de 242 personas. Para la recolección de datos se aplicó la técnica de la entrevista, utilizando dos instrumentos: la escala del estilo de vida y el cuestionario semiestructurado sobre factores biosocioculturales de la persona adulta; instrumentos garantizados en su validez y confiabilidad mediante la prueba de Alpha de Crombach. ${ }^{5,6}$

\section{RESULTADOS}

Tabla 01

ESTILO DE VIDA DEL ADULTO MAYOR DE LA URBANIZACIÓN EL CARMEN - CHIMBOTE, 2012

\begin{tabular}{lcc}
\hline Estilo de vida & $\mathbf{N}^{\circ}$ & $\%$ \\
\hline Saludable & 140 & 57,9 \\
No saludable & 102 & 42,1 \\
\hline Total & 242 & 100,0 \\
\hline Fuente: Escala de estilo de vida elaborada por: Walker, Sechristy Pender, modificado por: \\
Díaz, R; Reyna, E; Delgado, R. aplicado a los adultos mayores de la urbanización "El \\
Carmen", febrero, 2012.
\end{tabular}

La tabla 01 reporta que el $57,9 \%$ de los adultos mayores tiene un estilo de vida saludable y el $42.1 \%$, un estilo de vida no saludable.

Tabla 02

FACTORES BIOSOCIOCULTURALES DEL ADULTO MAYOR

\begin{tabular}{lr}
\hline \multicolumn{1}{c}{ FACTORES BIOLÓGICOS } \\
\hline Sexo & N \\
Masculino & 112 \\
Femenino & 130 \\
Total & 242 \\
\multicolumn{1}{c}{ FACTORES CULTURALES } \\
\hline Grado de instrucción \\
Analfabeto(a) \\
Primaria incompleta \\
Primaria completa & 15 \\
Secundaria incompleta & 48 \\
Secundaria completa & 59 \\
Superior incompleta & 46 \\
Superior completa & 28 \\
Total & 30 \\
& 16 \\
\hline
\end{tabular}


Tabla 02 (Cont.)

\begin{tabular}{lr} 
Religión & $\mathbf{N}$ \\
Católica & 205 \\
Evangélica & 33 \\
Otras & 4 \\
Total & 242 \\
\hline \multicolumn{1}{c}{ FACTORES SOCIALES } \\
Estado civil & $\mathbf{N}$ \\
Casado (a) & 177 \\
Viudo & 65 \\
Total & 242 \\
Ocupación & $\mathbf{N}$ \\
Obrero(a) & 40 \\
Ama de casa & 128 \\
Otras & 74 \\
Total & 242 \\
Ingreso económico (soles) & $\mathbf{N}$ \\
Menos de 100 & 44 \\
De 100 a 599 & 31 \\
De 600 a 1000 & 117 \\
Más de 1000 & 50 \\
Total & 242 \\
\hline
\end{tabular}

Fuente: Cuestionario de factores biosoculturales, elaborado por: Díaz, R; Reyna, E; Delgado,

R., aplicado a los adultos mayores de la urbanización “El Carmen”, febrero, 2012.

La tabla 02, sobre los factores biosocioculturales de los adultos mayores reporta que, el 53,7\% son del sexo femenino; el 24,4\% tienen grado de instrucción primaria completa; el $84,7 \%$ son católicos; el 73,1\% es de estado civil casado; el 52,9\% son amas de casa y el 48,3\% tiene un ingreso económico de 600 a 1000 nuevos soles.

La tabla 03 reporta que el $31,4 \%$ de los adultos mayores de sexo femenino presenta un estilo de vida saludable y el $22,3 \%$, un estilo de vida no saludable. Por otro lado, el $26,4 \%$ de adultos mayores de sexo masculino presenta estilo de vida saludable y el $19.8 \%$, un estilo de vida no saludable.

La tabla 04 reporta que el $15,7 \%$ de los adultos mayores tiene grado de instrucción secundaria incompleta, con un estilo de vida saludable; por otro lado, el 47,5\% de los adultos mayores profesa la religión católica, con un estilo de vida saludable. 
Tabla 03

FACTORES BIOLÓGICOS Y ESTILO DE VIDA DE LOS ADULTOS MAYORES

\begin{tabular}{|c|c|c|c|c|c|c|}
\hline \multirow{3}{*}{ Sexo } & \multicolumn{6}{|c|}{ Estilo de vida } \\
\hline & \multicolumn{2}{|c|}{ Saludable } & \multicolumn{2}{|c|}{ No saludable } & \multicolumn{2}{|c|}{ Total } \\
\hline & $\mathrm{n}$ & $\%$ & $\mathbf{n}$ & $\%$ & $\mathbf{n}$ & $\%$ \\
\hline Masculino & 64 & 26,4 & 48 & 19,8 & 112 & 46,3 \\
\hline Femenino & 76 & 31,4 & 54 & 22,3 & 130 & 53,7 \\
\hline Total & 140 & 57,9 & 102 & 42,1 & 242 & 100,0 \\
\hline
\end{tabular}

Fuente: Cuestionario de factores biosocioculturales elaborado por: Díaz, R; Reyna, E; Delgado, R. y Escala de estilo de vida elaborada por: Walker, Sechrist y Pender, modificado por: Díaz, R; Reyna, E; Delgado, R., aplicado los adultos mayores de la urbanización "El Carmen”, febrero, 2012.

Tabla 04

FACTORES CULTURALES Y ESTILO DE VIDA DE

LOS ADULTOS MAYORES

\begin{tabular}{|c|c|c|c|c|c|c|}
\hline & \multicolumn{6}{|c|}{ Estilo de vida } \\
\hline & \multicolumn{2}{|c|}{ Saludable } & \multicolumn{2}{|c|}{ No saludable } & \multicolumn{2}{|c|}{ Total } \\
\hline & $\mathrm{n}$ & $\%$ & $\mathbf{n}$ & $\%$ & $\mathbf{n}$ & $\%$ \\
\hline \multicolumn{7}{|l|}{ Grado de instrucción } \\
\hline Analfabeto(a) & 9 & 3,7 & 6 & 2,5 & 15 & 6,2 \\
\hline Primaria incompleta & 21 & 8,7 & 27 & 11,2 & 48 & 19,8 \\
\hline Primaria Completa & 28 & 11,6 & 31 & 12,8 & 59 & 24,4 \\
\hline Secundaria incompleta & 38 & 15,7 & 8 & 3,3 & 46 & 19,0 \\
\hline Secundaria completa & 21 & 8,7 & 7 & 2,9 & 28 & 11,6 \\
\hline Superior incompleta & 15 & 6,2 & 15 & 6,2 & 30 & 12,4 \\
\hline Superior completa & 8 & 3,3 & 8 & 3,3 & 16 & 6,6 \\
\hline Total & 140 & 57,9 & 102 & 42,1 & 242 & 100,0 \\
\hline
\end{tabular}

Pruebas de Chi-cuadrado

$$
\begin{gathered}
\chi^{2}=22,660 ; 6 \mathrm{gl} \mathrm{y} \mathrm{p}=0,001 \\
\mathrm{p}<0,05
\end{gathered}
$$

Si existe relación entre las variables. 
Tabla 04 (Cont.)

\begin{tabular}{lrrrrrr}
\hline & \multicolumn{9}{c}{ Estilo de vida } \\
\cline { 2 - 7 } & \multicolumn{2}{c}{ Saludable } & \multicolumn{2}{c}{ No saludable } & \multicolumn{2}{c}{ Total } \\
\cline { 2 - 8 } & \multicolumn{1}{c}{$\mathrm{n}$} & $\%$ & $\mathbf{n}$ & $\%$ & $\mathbf{n}$ & $\%$ \\
\hline Religión & & & & & & \\
Católico(a) & 115 & 47,5 & 90 & 37,2 & 205 & 84,7 \\
Evangélico(a) & 24 & 9,9 & 9 & 3,7 & 33 & 13,6 \\
Otras & 1 &, 4 & 3 & 1,2 & 4 & 1,7 \\
Total & 140 & 57,9 & 102 & 42,1 & 90 & 100,0 \\
\hline
\end{tabular}

Pruebas de Chi-cuadrado

$$
\begin{gathered}
\chi^{2}=5,024 ; 2 \mathrm{gl} \text { y } \mathrm{p}=0,081 \\
\mathrm{p}>0,05
\end{gathered}
$$

No existe relación entre las variables.

Fuente: Cuestionario de factores biosocioculturales elaborado por: Díaz, R; Reyna, E; Delgado, R. y Escala de estilo de vida elaborada por: Walker, Sechrist y Pender, modificado por: Díaz, R; Reyna, E; Delgado, R., aplicado a los adultos mayores de la urbanización "El Carmen”, febrero, 2012.

Tabla 05

FACTORES SOCIALES Y ESTILO DE VIDA DE LOS ADULTOS MAYORES

\begin{tabular}{lrrrrrr}
\hline & \multicolumn{9}{c}{ Estilo de vida } \\
\cline { 2 - 8 } & \multicolumn{2}{c}{ Saludable } & \multicolumn{2}{c}{ No saludable } & \multicolumn{2}{c}{ Total } \\
\cline { 2 - 8 } & \multicolumn{1}{c}{$\mathrm{n}$} & $\%$ & $\mathbf{n}$ & $\%$ & $\mathbf{n}$ & $\%$ \\
\hline Estado civil & & & & & & \\
Casado(a) & 101 & 41,7 & 76 & 31,4 & 177 & 73,1 \\
Viudo(a) & 39 & 16,1 & 26 & 10,7 & 65 & 26,9 \\
Total & 140 & 57,9 & 102 & 42,1 & 242 & 100,0 \\
\hline
\end{tabular}

Pruebas de Chi-cuadrado

$$
\begin{gathered}
\chi^{2}=0,168 ; 1 \mathrm{gl} \text { y } \mathrm{p}=0,682 \\
\mathrm{p}>0,05
\end{gathered}
$$

No existe relación entre las variables. 
Tabla 05 (Cont.)

\begin{tabular}{lrrrrrr}
\hline & \multicolumn{9}{c}{ Estilo de vida } \\
\cline { 2 - 7 } & \multicolumn{2}{c}{ Saludable } & \multicolumn{2}{c}{ No saludable } & \multicolumn{2}{c}{ Total } \\
\cline { 2 - 7 } Ocupación & \multicolumn{1}{c}{$\mathrm{n}$} & $\%$ & $\mathbf{n}$ & $\%$ & $\mathbf{n}$ & $\%$ \\
Obrero(a) & 22 & 9,1 & 18 & 7,4 & 40 & 16,5 \\
Ama de casa & 75 & 31,0 & 53 & 21,9 & 128 & 52,9 \\
Otros & 43 & 17,8 & 31 & 12,8 & 74 & 30,6 \\
Total & 140 & 50,0 & 102 & 42,1 & 242 & 100,0 \\
\hline
\end{tabular}

Pruebas de Chi-cuadrado

$\chi^{2}=0,164 ; 2 \mathrm{gl} \mathrm{y} \mathrm{p}=0,921$

$\mathrm{p}>0,05$

No existe relación entre las variables.

\begin{tabular}{lrrrrrr}
\hline & \multicolumn{9}{c}{ Estilo de vida } \\
\cline { 2 - 7 } & \multicolumn{2}{c}{ Saludable } & \multicolumn{2}{c}{ No saludable } & \multicolumn{2}{c}{ Total } \\
\cline { 2 - 8 } & \multicolumn{1}{c}{$\mathrm{n}$} & $\mathbf{\%}$ & $\mathbf{n}$ & $\mathbf{\%}$ & $\mathbf{n}$ & $\%$ \\
\hline \multicolumn{2}{l}{ Ingresos económicos (soles) } & & & & & \\
Menos de 100 & 26 & 10,7 & 18 & 7,4 & 44 & 18,2 \\
$100-599$ & 12 & 5,0 & 19 & 7,9 & 31 & 12,8 \\
$600-1000$ & 69 & 28,5 & 48 & 19,8 & 117 & 48,3 \\
Más de 1000 & 33 & 13,6 & 17 & 7,0 & 50 & 20,7 \\
Total & 140 & 57,9 & 102 & 42,1 & 242 & 100,0 \\
\end{tabular}

Pruebas de Chi-cuadrado

$\chi^{2}=6,108 ; 3 \mathrm{gl} \mathrm{y} \mathrm{p}=0,106$

$\mathrm{p}>0,05$

No existe relación entre las variables.

Fuente: Cuestionario de factores biosocioculturales elaborado por: Díaz, R; Reyna, E; Delgado, R. y Escala de estilo de vida elaborada por: Walker, Sechrist y Pender, modificado por: Díaz, R; Reyna, E; Delgado, R., aplicado a los adultos mayores de la urbanización “El Carmen”, febrero, 2012. 
Finalmente, la tabla 05 reporta que el $41,7 \%$ de los adultos mayores es de estado civil casado, con un estilo de vida saludable; el $31 \%$ son amas de casa, con un estilo de vida saludable; el 28,5\% tiene un ingreso económico de 600 a 1000 nuevos soles, y cuenta con un estilo de vida saludable.

\section{DISCUSIÓN}

En la tabla 01 se muestra que del 100\% (242) de personas adultas mayores que participaron en el estudio, el 57,9\% (140) tiene un estilo de vida saludable y un $42,1 \%$ (102), un estilo de vida no saludable.

Los resultados de la presente investigación se relacionan con los encontrados por Felipe, L. ${ }^{7}$ en su tesis de investigación sobre estilos de vida y factores biosocioculturales del adulto mayor en el A.H. Villa Magisterial I y II - Nuevo Chimbote, donde encontró que el $88 \%$ tiene un estilo de vida saludable y el $12 \%$, un estilo de vida no saludable.

El estilo de vida es la expresión de lo social a través de la actividad individual de la personalidad. Todas las personas realizan actividades del modo de vida; pero la personalidad de cada individuo le otorga sentido a determinadas actividades o aspectos de ellas, las jerarquiza y al ejecutarlas les incorpora características personales, resultando un conjunto de actividades condicionadas por la personalidad, por lo que constituye un estilo de vida personal. ${ }^{8}$

En la tabla 02 se muestra la distribución porcentual de los factores biosocioculturales de los adultos mayores participantes en la presente investigación que residen la urbanización El Carmen. En los factores biológicos se observa que el mayor porcentaje de personas adultas mayores en un 53,7\% (130) son del sexo femenino. Con respecto a los factores culturales, el 24,4\% (59) de las personas adultas tiene primaria completa y un $84,7 \%$ (205) son católicos. Referente a los factores sociales, el 73,1\% (177) es casado; el 52,9\% (128) son amas de casa y un 48,3\% (117) tiene un ingreso económico de 600 a 1000 nuevos soles.

Estos resultados se relacionan en algunos indicadores con los obtenidos por Salome, $\mathrm{P} .{ }^{10}$ en su tesis de investigación Atención al anciano en el centro del adulto mayor de Essalud Tacna, donde encontró que el 65\% de los adultos mayores son de sexo femenino, y en cuanto al estado civil, el $75 \%$ son casados.

Asimismo, en los adultos mayores de la urbanización El Carmen existe mayor porcentaje de mujeres, debido a que existen viudas que viven con sus hijos(as). 
El grado de instrucción es un factor que garantiza la salud, ya que las características y accesibilidad a la educación pueden ayudar a la gente a encarar mejor el medio en que vive (para aumentar sus ingresos, para protegerse mejor contra los riesgos y para modificar su comportamiento en beneficio de la salud). ${ }^{11}$

Los adultos mayores de la urbanización El Carmen tienen, en mayor porcentaje, grado de instrucción de primaria completa, debido a que dejaban los estudios para trabajar y formar un hogar a temprana edad. Muchos de estos adultos mayores provienen de la zona de la sierra y al llegar a Chimbote no pudieron continuar sus estudios.

La mayoría de las amas de casa tiene una percepción positiva del trabajo remunerado porque les permite contar con sus propios ingresos, desarrollarse como persona, salir de la rutina y aumentar el presupuesto familiar. No obstante, el costo asociado a dejar solos a los hijos y descuidar la casa es muy alto. Muchas de ellas se ven obligadas a permanecer como amas de casa. ${ }^{12}$

Las amas de casa de la urbanización El Carmen, viven al cuidado del hogar, de sus esposos cuando llegan de trabajar o también cuando sus esposos son jubilados; de igual manera, manifiestan tener la capacidad adecuada para seguir atendiendo a sus esposos.

En relación con el ingreso económico, un gran porcentaje de los adultos mayores cuenta con un ingreso de 600 a 1000 nuevos soles mensuales, porque reciben pensión mensual de su jubilación, lo que les permite vivir adecuadamente.

En la tabla 03 se muestra que el $31,4 \%$ de los adultos mayores son del sexo femenino con un estilo de vida saludable y el $22,3 \%$ también son del sexo femenino; pero presentan un estilo de vida no saludable. De igual manera, el 26,4 \% de adultos mayores de sexo masculino presentan un estilo de vida saludable y el $19,8 \%$, un estilo no saludable

El sexo es el sistema compuesto por los órganos destinados a cumplir las funciones de la reproducción para conservar y perpetuar la especie y además constituye un factor determinante para establecer las diferencias físicas y psíquicas del varón y la mujer. ${ }^{13}$

Sin embargo, el ser masculino o femenino (varón o mujer) no va a determinar que los adultos opten por estilos de vida saludables o no, sino que ellos mismos van a determinar sus hábitos, los que los llevarán a la conservación de su salud. Esto queda demostrado con los resultados que determinan que el estilo de vida no se relaciona estadísticamente con los factores biológicos en los adultos mayores. 
Según Flores, L. y Hernández, L. en Sanabria, P. y col..$^{14}$, el estilo de vida se va conformando a lo largo de la vida del sujeto, pudiéndose afirmar que se trata de un proceso de aprendizaje, ya sea por asimilación o imitación de modelos familiares, de grupos formales o informales, pues es natural que las personas tiendan a imitar modelos de patrones formales o de patrones formales $o$ informales. Es natural también que las personas tiendan a imitar los modelos sociales promovidos en los medios de comunicación social. El estilo de vida se puede formar no solo espontáneamente, sino también por los resultados de las decisiones conscientes de quienes aspiran a perfeccionarse o a perfeccionar la sociedad. Representa, asimismo, un mundo complejo, fruto de los factores ambientales, personales y sociales, que convergen no solo del presente, sino también de la historia interpersonal.

En la tabla 04 se muestra la relación entre los factores biosocioculturales y el estilo de vida. Al relacionar la religión con el estilo de vida utilizando el estadístico Chi-cuadrado de independencia $\left(\chi^{2}=5,024 ; 2 \mathrm{gl} \mathrm{y} \mathrm{p}=0,081 \mathrm{p}>0,05\right)$, encontramos que no existe relación estadísticamente significativa entre ambas variables. Se muestra que existe relación entre el factor biosociocultural grado de instrucción y estilo de vida utilizando el estadístico Chi-cuadrado de independencia $\left(\chi^{2}=22,660 ; 6\right.$ gl y $\left.\mathrm{p}=0,001 \mathrm{p}<0,05\right)$.

Los resultados obtenidos se relacionan con los encontrados por Pujay, Y. ${ }^{5}$, en su estudio sobre Estilos de vida y factores biosocioculturales del adulto mayor en la Clínica Geriátrica de San Isidro Labrador en Ate - Lima, quien reportó que no existe relación estadísticamente significativa entre estilo de vida y los factores culturales.

El grado de instrucción se define como el último año escolar cursado por la persona, según el cual puede demostrar sus capacidades cognitivas conceptuales, actitudinales y procedimentales. ${ }^{16}$

Aunque los medios de comunicación afirman que existe alguna relación entre los factores culturales y el estilo de vida, en general, las situaciones que se presentan en relación con el cuidado de la salud, donde el nivel cultural alto es el factor protector e influye en las conductas saludables y la religiosidad evita o restringe algunas conductas no saludables como el consumo de sustancias nocivas. En este estudio los resultados y la prueba estadística confirman que la variable factores culturales no tienen relación con el estilo de vida del adulto mayor.

La mayoría de los adultos mayores de la población estudiada tiene grado de instrucción de primaria completa, ya que tenían el deber de trabajar a temprana edad y eran el sustento de su familia. Por otro lado, la presente investiga- 
ción reporta que hay adultos mayores que aún persisten con los estilos de vida no saludables. Ello se debe a que hay costumbres arraigadas que se practican de generación en generación y son muy difíciles de desterrar o cambiar aun cuando reciban la información correcta, por lo que se hace necesario que tomen conciencia en su actuación para evitar enfermarse.

En la tabla 05 se muestra la relación entre los factores culturales y el estilo de vida. Al relacionar el estado civil con el estilo de vida utilizando el estadístico Chi-cuadrado de independencia $\left(\chi^{2}=0,168 ; 1 \mathrm{gl} \mathrm{y} \mathrm{p}=0,682 \mathrm{p}>0,05\right)$, encontramos que no existe relación estadísticamente significativa entre ambas variables. De igual modo, en la relación entre la ocupación y estilo de vida, se encontró que no existe relación estadísticamente significativa $\left(\chi^{2}=0,164 ; 2 \mathrm{gl}\right.$ y $\mathrm{p}=0,921)$ entre la religión y el estilo de vida. También se muestra relación entre los ingresos económicos y estilos de vida, encontrando que no existe relación estadísticamente significativa $\left(\chi^{2}=6,108 ; 3 \mathrm{gl} \mathrm{y} \mathrm{p}=0,106 \mathrm{p}>0,05\right)$.

Los resultados coinciden con lo obtenido por Felipe, L. ${ }^{7}$, quien investigó el estilo de vida y los factores biosocioculturales del adulto mayor en el A.H. Villa Magisterial, I y II etapa, Nuevo Chimbote, y concluye que los factores culturales no tienen relación con el estilo de vida.

Por otro lado, los resultados obtenidos en esta investigación difieren en algunos indicadores con lo encontrado por Luciano, C. y Reyes, S. ${ }^{17}$ en su estudio Estilos de vida y factores biosocioculturales de la persona adulta del A.H. Tres Estrellas en Chimbote, donde se concluye que existe relación estadísticamente significativa entre el estilo de vida y la religión.

Los resultados encontrados se relacionan también con los encontrados por Mujica, K. ${ }^{8}$, en su estudio El estilo de vida y factores biosocioculturales del adulto mayor en Nuevo Chimbote, donde concluye que no existe relación estadísticamente significativa entre el estilo de vida y los factores sociales.

De igual modo, los resultados encontrados en esta investigación se relacionan con lo encontrado por López, S. y Alva, M. ${ }^{12}$, quienes estudiaron los estilos de vida y los factores biosocioculturales de la persona adulta en Nuevo Chimbote, concluyendo que no existe relación estadísticamente significativa entre el estilo de vida con el estado civil, ocupación e ingreso económico.

El estilo de vida se origina en las motivaciones e intereses que tienen las personas. Algunos autores mencionan que también influyen la formación que estos han tenido en su hogar y las experiencias sobre el proceso saludenfermedad. Los factores sociales como la ocupación, el estado civil y el ingreso económico, no determinan el estilo de vida que presentan las personas. 


\section{CONCLUSIONES}

1. Más de la mitad de los adultos mayores presenta estilos de vida saludable y en porcentaje considerable tienen estilos de vida no saludable.

2. Respecto a los factores biosocioculturales, más de la mitad son de sexo femenino; menos de la mitad presenta grado de instrucción primaria completa e incompleta y percibe de 600 a 1000 soles mensuales. La mayoría es de religión católica y de estado civil casado; poco más de la mitad son amas de casa.

3. Sí existe relación estadísticamente significativa entre el estilo de vida y el factor cultural Grado de instrucción en los adultos mayores.

4. No existe relación estadísticamente significativa entre los estilos de vida y los factores biosocioculturales en cuanto se refieren a edad, sexo, estado civil, ocupación e ingreso económico.

\section{REFERENCIAS BIBLIOGRÁFICAS}

1 Soto L. Estilos de vida y calidad de vida. [Artículo en internet]. México. [citado 2010 Ago. 10]. Disponible en URL: http://www.mitecnologico.com/Main/EstilosDeVidaYCalidadDeVida.

2 Wikipedia. Estilo de vida. [Artículo en internet]. 2010 [citado 2010 Ago. 10]. Disponible en URL: http://es.wikipedia.org/wiki/Estilo_de_vida.

3 Hill D, Nishida C. Un enfoque basado en el ciclo vital para la dieta, nutrición y prevención de enfermedades crónicas. [Portada en internet].2007. [Citado 2010 Ago. 11]. Disponible en URL: http://www.bago.com/BagoArg/Biblio/nutriweb184.htm

4 Puesto de SAlud "Túpac Amaru". Sistema de vigilancia comunal - Consolidado total de las fichas de la urbanización "El Carmen" 2011(documento del puesto de salud "Túpac Amaru") Chimbote. Noviembre, 2011.

5 Nivel de instrucción. [Portal de internet]. 2004. [Citado 2010 Set. 5]. [alrededor de 1 pantalla]. Disponible en URL: http://www.eustat.es/documentos/idioma_c/opt_0/tema_303/elem_2376/ definicion.html

6 HERnÁNDEZ R. Metodología de la investigación. [Artículo en internet]. México: Editorial Mc Graw Hill; 2003. [Citado 2010 Ago. 15]. Disponible en URL: http://www.metabase.net/docs/ unibe/ 03624.html.

7 FigueroA D. Seguridad alimentaria y familiar. [Revista en internet]. 2003. [Citado 2010 Ago. 27]. [alrededor de 1 pantalla]. Disponible en URL: http://www.respyn.uanl.mx/iv/2/ensayos/ seguridad_alimentaria.htm

8 Mendoza S. Factores biosocioculturales y auto cuidado en el adulto diabético del Hospital III. Essalud. [tesis en interne] Chimbote; 2007. [Citado 2010 Ago. 15]. Disponible en URL: http://www.uladech.edu.pe/webuladech/demi/compendio_enfermeria.htm

9 PALANCO K, Ramos L. Estilos de vida y factores biosocioculturales de la persona adulta de la comunidad Señor de Palacios. Ayacucho [tesis para optar el título de enfermería]. Piura, Perú: Universidad Católica Los Ángeles de Chimbote; 2009. 
10 Tineo L. Estilos de vida de los Pacientes del Programa de Hipertensión Arterial del Hospital II Jorge Reátegui Delgado Essalud [tesis para optar el titulo de enfermería]. Piura, Perú: Universidad Nacional de Piura; 2006.

11 Wikipedia. La enciclopedia libre. salud. [Artículo de internet]. 2010 [Citado 2010 Ago. 19]. [1 pantalla]. Disponible en URL: http://es.wikipedia.org/wiki/Salud

12 OM-C salud. Un estilo de vida saludable [Articulo en internet]. México. [Citado 2010 Ago. 18]. [1 pantalla]. Disponible en URL: http://www.omcsalud.com/contenido/articulos/un-estilo-de-vidasaludable.html

13 ORganización Panamericana de la SAlud (OPS), Organización Mundial de la Salud (OMS) Y Asociación Mundial de Sexología. Promoción de la Salud Sexual; Recomendaciones para la acción. [Documento en internet]. Guatemala; 2000. [1 pantalla]. Disponible en URL: http://www.amssac.org/biblioteca\%20sexualidad\%20conceptos.htm

14 Wikipedia. Religión. [Artículo en internet]. 2010 [Citado 2010 Set 09]. Disponible en URL: http://es.wikipedia.org/wiki/Religión

15 Definición de ocupación. [Portada en internet]. 2008. [Citado 2010 Set 09]. Disponible desde el URL: http://definicion.de/ocupacion/

16 Wikipedia. salario. [Artículo en internet]. 2010 [Citado 2010 Set 09.]. Disponible en URL: http://es.wikipedia.org/wiki/Salario

17 GoNZÁLEZ M. Diseños experiméntales de investigación. [Monografía en internet]. España; 2010. [Citado 2010 Set 12]. Disponible en URL: http://www.monografias.com/trabajos10/cuasi/ cuasi.shtml 